\title{
ON THE ALMOST PERIODIC AT INFINITY FUNCTIONS FROM HOMOGENEOUS SPACES
}

\begin{abstract}
We consider homogeneous spaces of functions defined on the real axis (or semi-axis) with values in a complex Banach space. We study the new class of almost periodic at infinity functions from homogeneous spaces. The main results of the article are connected to harmonic analysis of those functions. We give four definitions of an almost periodic at infinity function from a homogeneous space and prove them to be equivalent. We also introduce the concept of a Fourier series with slowly varying at infinity coefficients (neither necessarily constant nor necessarily having a limit at infinity). It is proved that the Fourier coefficients of almost periodic at infinity function from a homogeneous space (not necessarily continuous) can be chosen continuous. Moreover, they can be extended on $\mathbb{C}$ to bounded entire functions of exponential type. Besides, we prove the summability of Fourier series by the method of Bochner-Fejer. The results were received with essential use of isometric representations and Banach modules theory.
\end{abstract}

Key words: almost periodic at infinity function, homogeneous space, Banach module, almost periodic vector, Fourier series

2010 Mathematical Subject Classification: 33E30, 43A60, $46 B 04$

1. Homogeneous function spaces. Let $X$ be a complex Banach space, End $X$ be a Banach algebra of bounded linear operators on $X$. Let $\mathbb{J}$ be either $\mathbb{R}_{+}=[0, \infty)$, or $\mathbb{R}=(-\infty, \infty)$.

By $L_{l o c}^{1}(\mathbb{J}, X)$ denote the space of Bochner measurable locally integrable on $\mathbb{J}$ (classes of) functions with values in $X$. By $S^{p}(\mathbb{J}, X), p \in[1, \infty)$, denote the Stepanov space of functions [15] $x \in L_{l o c}^{1}(\mathbb{J}, X)$ with the following norm:

$$
\|x\|_{S^{p}}=\sup _{s \in \mathbb{J}}\left(\int_{0}^{1}\|x(s+t)\|_{X}^{p} d t\right)^{1 / p}, \quad p \in[1, \infty) .
$$

(c) Petrozavodsk State University, 2018 
Stepanov spaces play an important role in studying differential equations in Banach spaces (see [4]).

Definition 1. A Banach space $\mathcal{F}(\mathbb{R}, X)$ of functions defined on $\mathbb{R}$ with values in a Banach space $X$ is called homogeneous, if the following conditions are satisfied:

(a) the space $\mathcal{F}(\mathbb{R}, X)$ is injectively (which means injectivity of the inclusion operator) and continuously embedded in $S^{1}(\mathbb{R}, X)$;

(b) a group of shift operators $S(t), t \in \mathbb{R}$, of the form

$$
(S(t) x)(s)=x(s+t), s, t \in \mathbb{R}, x \in \mathcal{F}(\mathbb{R}, X),
$$

is defined on $\mathcal{F}(\mathbb{R}, X)$;

(c) for any functions $f \in L^{1}(\mathbb{R}), x \in \mathcal{F}(\mathbb{R}, X)$ their convolution defined by

$$
(f * x)(t)=\int_{\mathbb{R}} f(\tau) x(t-\tau) d \tau=\int_{\mathbb{R}} f(\tau)(S(-\tau) x)(t) d \tau, t \in \mathbb{R},
$$

belongs to $\mathcal{F}(\mathbb{R}, X)$ and satisfies the condition $\|f * x\| \leqslant C\|f\|_{1}\|x\|$ for some $C \geqslant 1$ (usually $C=1$ );

(d) the inclusion $\varphi x \in \mathcal{F}(\mathbb{R}, X)$ holds for any $x \in \mathcal{F}(\mathbb{R}, X)$ and any infinitely differentiable function $\varphi \in C_{b}(\mathbb{R})$ with compact support $\operatorname{supp} \varphi ;$ moreover, inequality $\|\varphi x\| \leqslant\|\varphi\|_{1}\|x\|$ holds and the mapping $t \mapsto \varphi S(t) x: \mathbb{R} \rightarrow \mathcal{F}(\mathbb{R}, X)$ is continuous.

By $\mathcal{F}_{0}(\mathbb{R}, X)$ we denote the least closed subspace of $\mathcal{F}(\mathbb{R}, X)$ containing all functions $\varphi x, x \in \mathcal{F}(\mathbb{R}, X)$, where $\varphi \in C_{b}(\mathbb{R}, X)$ is infinitely differentiable and its support $\operatorname{supp} \varphi$ is a compact set.

Definition 2. A Banach space $\mathcal{F}\left(\mathbb{R}_{+}, X\right)$ of functions from $S^{1}\left(\mathbb{R}_{+}, X\right)$ is called homogeneous, if a homogeneous space $\mathcal{F}(\mathbb{R}, X)$ associated with $\mathcal{F}\left(\mathbb{R}_{+}, X\right)$ exists, such that for any function $x \in \mathcal{F}\left(\mathbb{R}_{+}, X\right)$ there is an extension $y \in \mathcal{F}(\mathbb{R}, X)$ with the following properties:

1) $y(t)=x(t)$ for all $t \in \mathbb{R}_{+}$;

2) $\|y\| \leqslant C\|x\|, C>0$

3) $y \in \mathcal{F}_{0}\left(\mathbb{R}_{-}, X\right)$;

4) $S(t) x \in \mathcal{F}\left(\mathbb{R}_{+}, X\right)$ for all $t \geqslant 0, x \in \mathcal{F}\left(\mathbb{R}_{+}, X\right)$; 
5) for any other extension $z \in \mathcal{F}(\mathbb{R}, X)$ with the properties 1)-4) the condition $y-z \in \mathcal{F}_{0}(\mathbb{R}, X)$ holds.

In this article we denote a homogeneous space by $\mathcal{F}(\mathbb{J}, X)$, while in case $X=\mathbb{C}$ the notation $\mathcal{F}(\mathbb{J})$ can be used. Denote a closed subspace of $\mathcal{F}(\mathbb{J}, X)$ defined by $\{x \in \mathcal{F}(\mathbb{J}, X)$ : function $t \mapsto S(t) x: \mathbb{J} \rightarrow \mathcal{F}(\mathbb{J}, X)$ is continuous $\}$ by $\mathcal{F}_{c}(\mathbb{J}, X)$.

Example 1. The following Banach spaces of functions defined on $\mathbb{J}$ with values in a Banach space $X$ are homogeneous. All of them are linear subspaces of $L_{l o c}^{1}(\mathbb{J}, X)$.

1) The spaces $L^{p}=L^{p}(\mathbb{J}, X), p \in[1, \infty)$, of Lebesgue measurable and integrable with power $p \in[1, \infty)$ (classes of) functions with the norm $\|x\|_{p}=\left(\int_{\mathbb{J}}\|x(t)\|_{X}^{p} d t\right)^{1 / p}, p \in[1, \infty)$.

Note that $\left(L^{p}(\mathbb{J}, X)\right)_{c}=L^{p}(\mathbb{I}, X), \quad\left(L^{p}(\mathbb{J}, X)\right)_{0}=L^{p}(\mathbb{J}, X)$.

2) The space $L^{\infty}=L^{\infty}(\mathbb{J}, X)$ of essentially bounded (classes of) functions with the following norm: $\|x\|_{\infty}=$ vrai $\sup _{t \in \mathbb{J}}\|x(t)\|_{X}$. Note that $\left(L^{\infty}(\mathbb{J}, X)\right)_{c}=C_{b, u}(\mathbb{J}, X)$.

3) Stepanov spaces $S^{p}=S^{p}(\mathbb{J}, X), p \in[1, \infty)$.

4) Wiener amalgam spaces $\left(L^{p}, l^{q}\right)=\left(L^{p}(\mathbb{J}, X), l^{q}(\mathbb{J}, X)\right), p \in[1, \infty)$, $q \in[1, \infty]$, (see [4]) of functions $x \in L_{\text {loc }}^{1}(\mathbb{J}, X)$ such that

$$
\|x\|_{p, q}=\left(\sum_{k \in \mathbb{Z}}\left(\int_{0}^{1}\|x(s+k)\|^{p} d s\right)^{q / p}\right)^{1 / q}<\infty, p, q \in[1, \infty) .
$$

The following equivalent norm can also be used:

$$
\|x\|_{p, q}=\sup _{t \in[0,1]}\left(\sum_{k \in \mathbb{Z}}\left(\int_{0}^{1}\|x(s+t+k)\|^{p} d s\right)^{q / p}\right)^{1 / q}<\infty, p, q \in[1, \infty) .
$$

$5)$ The space $C_{b}=C_{b}(\mathbb{J}, X)$ of bounded continuous functions with the norm $\|x\|_{\infty}=\sup _{t \in \mathbb{J}}\|x(t)\|_{X}, x \in C_{b}(\mathbb{J}, X)$. Note that $C_{b}(\mathbb{J}, X)$ is a closed subspace of $L^{\infty}(\mathbb{J}, X)$ and $\left(C_{b}(\mathbb{J}, X)\right)_{c}=C_{b, u}(\mathbb{J}, X)$, $\left(C_{b}(\mathbb{J}, X)\right)_{0}=C_{0}(\mathbb{J}, X)$.

6) The subspace $C_{b, u}=C_{b, u}(\mathbb{J}, X)$ of $C_{b}(\mathbb{J}, X)$ of uniformly continuous functions from $C_{b}$. Note that $\left(C_{b, u}(\mathbb{J}, X)\right)_{c}=C_{b, u}(\mathbb{J}, X)$ and $\left(C_{b, u}(\mathbb{J}, X)\right)_{0}=C_{0}(\mathbb{J}, X)$. 
7) The subspace $C_{0}=C_{0}(\mathbb{J}, X)$ of $C_{b, u}(\mathbb{J}, X)$ of continuous functions, vanishing at infinity. These functions satisfy the condition $\lim _{|t| \rightarrow \infty}\|x(t)\|=0, t \in \mathbb{J}$.

8) The subspace $C_{s l, \infty}=C_{s l, \infty}(\mathbb{J}, X)$ of $C_{b, u}(\mathbb{J}, X)$ of continuous functions slowly varying at infinity. These functions satisfy the condition $\lim _{|t| \rightarrow \infty}\|x(t+\tau)-x(t)\|=0, t, \tau \in \mathbb{J}($ see $[12,17-19])$.

9) The subspace $C_{\omega, \infty}=C_{\omega, \infty}(\mathbb{J}, X)$ of $C_{b, u}(\mathbb{J}, X)$ of continuous $\omega$-periodic at infinity functions, $\omega \in \mathbb{R}_{+}$. These functions satisfy the condition $\lim _{|t| \rightarrow \infty}\|x(t+\omega)-x(t)\|=0, t \in \mathbb{J}$ (see [12,17-19]).

10) The subspace $A P_{\infty}=A P_{\infty}(\mathbb{J}, X)$ of $C_{b, u}(\mathbb{I}, X)$ of continuous almost periodic at infinity functions (see $[3,4])$.

11) Subspaces $C^{k}=C^{k}(\mathbb{J}, X), k \in \mathbb{N}$, of $k$ times continuously differentiable functions with bounded $k$-th derivative and the norm $\|x\|_{(k)}=\|x\|_{\infty}+\left\|x^{(k)}\right\|_{\infty}$.

12) Hölder spaces $C^{k, \alpha}=C^{k, \alpha}(\mathbb{J}, X), k \in \mathbb{N} \cup\{0\}, \alpha \in(0,1]$,

$$
\begin{aligned}
C^{k, \alpha} & =\left\{x \in C^{k}:\left\|x^{(k)}\right\|_{C^{0, \alpha}}=\sup _{t \neq s \in \mathbb{J}} \frac{\left|x^{(k)}(t)-x^{(k)}(s)\right|}{|t-s|^{\alpha}}<\infty\right\}, \\
\|x\|_{C^{k, \alpha}} & =\|x\|_{C^{k}}+\left\|x^{(k)}\right\|_{C^{0, \alpha}} .
\end{aligned}
$$

13) The subspace $\mathbb{V}=\mathbb{V}(\mathbb{J}, X)$ of functions from $L^{\infty}(\mathbb{J}, X)$ with bounded variation $\|x\|_{\mathbb{V}}=\sup _{t \in \mathbb{J}} V_{t}^{t+1}(x)+\sup _{t \in \mathbb{J}}\|x\|_{X}$ used as a norm.

Definition 1 implies that each of the mentioned homogeneous spaces $\mathcal{F}(\mathbb{R}, X)$ is endowed with the structure of a Banach $L^{1}(\mathbb{R})$-module on using the convolution (2), where $S$ is a group of shifts defined by (1). Thus, there is an opportunity to use some notions and results of the theory of Banach $L^{1}(\mathbb{R})$-modules given below. In particular, the spaces $\mathcal{F}_{c}(\mathbb{R}, X)$ coincide with the spaces of $S$-continuous vectors (see Definition 3 ).

2. Almost periodic vectors from Banach $L^{1}(\mathbb{R})$-modules. Let $\mathcal{X}$ be a complex Banach space and End $\mathcal{X}$ be a Banach algebra of linear operators on $\mathcal{X}$. By $L^{1}(\mathbb{R})$ we denote the algebra of complex Lebesgue's measurable (classes of) functions summable on $\mathbb{R}$ with convolution as the multiplication: $(f * g)(t)=\int_{\mathbb{R}} f(t-s) g(s) d s, t \in \mathbb{R}, f, g \in L^{1}(\mathbb{R})$.

We endow $\mathcal{X}$ with the structure of a non-degenerate Banach $L^{1}(\mathbb{R})$ module (see $[5,10,14]$ ) associated with some bounded isometric representation $T: \mathbb{R} \rightarrow$ End $\mathcal{X}$. It means that the following properties hold. 
Assumption 1. A Banach $L^{1}(\mathbb{R})$-module $\mathcal{X}$ fulfills the conditions below:

1) if $f x=0$ for every function $f \in L^{1}(\mathbb{R})$, then the vector $x \in \mathcal{X}$ vanishes (a non-degeneracy property of $\mathcal{X}$ );

2) for every $f \in L^{1}(\mathbb{R})$ and $x \in \mathcal{X}$ the following equations hold:

$$
T(t)(f x)=(T(t) f) x=f(T(t) x), t \in \mathbb{R},
$$

the module structure on $\mathcal{X}$ is associated with the representation $T$ : $\mathbb{R} \rightarrow$ End $\mathcal{X}$.

If $T: \mathbb{R} \rightarrow$ End $\mathcal{X}$ is a strongly continuous isometric representation, then the formula

$$
T(f) x=f x=\int_{\mathbb{R}} f(t) T(-t) x d t, f \in L^{1}(\mathbb{R}), x \in \mathcal{X},
$$

endows $\mathcal{X}$ with the structure of a Banach $L^{1}(\mathbb{R})$-module satisfying the conditions of Assumption 1; this structure is associated with the representation $T$.

Remark 1. Associate a unique representation $T: \mathbb{R} \rightarrow$ End $\mathcal{X}$ (see [10]) with every non-degenerate Banach $L^{1}(\mathbb{R})$-module $\mathcal{X}$. In order to emphasise this, sometimes the notation $(\mathcal{X}, T)$ is used.

The theory of Banach $L^{1}(\mathbb{R})$-modules was constructed in [7] and studied in $[1,2,5,6,9,10,14]$.

Definition 3. A vector from a Banach $L^{1}(\mathbb{R})$-module $(\mathcal{X}, T)$ is called continuous (with respect to the representation $T$ ) or $T$-continuous if a function $\varphi_{x}: \mathbb{R} \rightarrow \mathcal{X}$ defined by $\varphi_{x}(t)=T(t) x, t \in \mathbb{R}$, is continuous for $t=0$ (hence, it is continuous on $\mathbb{R}$ ).

A set of all continuous vectors from a Banach $L^{1}(\mathbb{R})$-module $\mathcal{X}$ denoted by $\mathcal{X}_{c}$ or $(\mathcal{X}, T)_{c}$ is a closed submodule in $\mathcal{X}$, i. e., $\mathcal{X}_{c}$ is a closed linear subspace in $\mathcal{X}$ invariant under shift operators $T(f), T(t)$ for $f \in L^{1}(\mathbb{R})$ and $t \in \mathbb{R}$.

Every homogeneous space $\mathcal{F}(\mathbb{R}, X)$ is endowed with the structure of a Banach $L^{1}(\mathbb{R})$-module using the convolution (2), where $S: \mathbb{R} \rightarrow \operatorname{End} \mathcal{F}(\mathbb{R}, X)$ is the group of shifts defined in (1). However, formula (2) does not define the structure of $L^{1}(\mathbb{R})$-module for $\mathcal{F}\left(\mathbb{R}_{+}, X\right)$. Nevertheless, the quotient space $\mathcal{F}(\mathbb{J}, X) / \mathcal{F}_{0}(\mathbb{J}, X)$ is endowed with this structure. 
Given a function $f$ from $L^{1}(\mathbb{R})$, the Fourier transform $\widehat{f}: \mathbb{R} \rightarrow \mathbb{C}$ is defined as

$$
\widehat{f}(\lambda)=\int_{\mathbb{R}} f(t) e^{-i \lambda t} d t, \lambda \in \mathbb{R} .
$$

Definition 4. The Beurling spectrum of a vector $x \in \mathcal{X}$ is the set of numbers $\Lambda(x)$ in $\mathbb{R}$ defined by

$$
\Lambda(x)=\left\{\lambda_{0} \in \mathbb{R}: f x \neq 0 \text { for every function } f \in L^{1}(\mathbb{R}) \text { with } \widehat{f}\left(\lambda_{0}\right) \neq 0\right\} .
$$

The definition implies that $\Lambda(x)=\mathbb{R} \backslash\left\{\mu_{0} \in \mathbb{R}\right.$ : there exists a function $f \in L^{1}(\mathbb{R})$ such that $\widehat{f}\left(\mu_{0}\right) \neq 0$ and $\left.f x=0\right\}$.

The Beurling spectrum of vectors in a Banach $L^{1}(\mathbb{R})$-module $\mathcal{X}$ has the following properties (see $[5,10])$ :

Lemma 1. For every $x \in \mathcal{X}$ and $f \in L^{1}(\mathbb{R})$ the following properties hold:

1) the set $\Lambda(x)$ is closed and $\Lambda(x)=\emptyset$ if and only if $x=0$;

2) $\Lambda(f x) \subseteq(\operatorname{supp} \widehat{f}) \cup \Lambda(x)$;

3) $f x=0$ when $(\operatorname{supp} \widehat{f}) \bigcap \Lambda(x)=\emptyset$ and $f x=x$ if the set $\Lambda(x)$ is compact and $\widehat{f}=1$ in its neighbourhood;

4) the set $\Lambda(x)$ is a singleton $\left(\left(\Lambda(x)=\left\{\lambda_{0}\right\}\right)\right)$ if and only if $x \neq 0$ and $T(t) x=e^{i \lambda_{0} t} x$ for $t \in \mathbb{R}$.

Remark 2. As we indicated above, every homogeneous space $\mathcal{F}(\mathbb{R}, X)$ is a Banach $L^{1}(\mathbb{R})$-module. If a function $x \in \mathcal{F}(\mathbb{R}, X)$ has the property $\Lambda(x)=\left\{\lambda_{0}\right\}$, then it can be represented as $x(t)=x_{0} e^{i \lambda_{0} t}$ for $t \in \mathbb{R}$, where $x_{0} \in X$.

Now let us introduce $\lambda_{0}$-nets, bounded approximate identities (BAIs), and invariant integrals (see $[2,5,6,10]$ ), which are essential for our study.

Definition 5. Let $\mathcal{U}$ be a directed set and $\lambda_{0} \in \mathbb{R}$. A bounded net $\left(f_{\alpha}, \alpha \in \mathcal{U}\right)$ of functions from $L^{1}(\mathbb{R})$ is called a $\lambda_{0}$-net if the conditions below hold:

1) $\widehat{f}_{\alpha}\left(\lambda_{0}\right)=1$ for all $\alpha \in \mathcal{U}$;

2) $\lim _{\alpha} f_{\alpha} * f=0$ for any function $f \in L^{1}(\mathbb{R})$ with the property $\widehat{f}\left(\lambda_{0}\right)=0$.

As an example of a $\lambda_{0}$-net from $L^{1}(\mathbb{R})$ one can consider the functions $g_{\alpha}(t)=f_{\alpha}(t) e^{i \lambda_{0} t}, \psi_{\alpha}(t)=\varphi_{\alpha}(t) e^{i \lambda_{0} t}, \alpha>0$, where 0-nets $\left(f_{\alpha}, \alpha>0\right)$ and $\left(\varphi_{\alpha}, \alpha>0\right)$ are defined by 


$$
\begin{gathered}
f_{\alpha}(t)=\left\{\begin{array}{ll}
\alpha e^{-\alpha t}, & t \geqslant 0, \\
0, & t<0,
\end{array} \quad \alpha>0,\right. \\
\varphi_{\alpha}(t)=\left\{\begin{array}{ll}
(2 \alpha)^{-1}, & t \in[-\alpha, \alpha], \\
0, & t \notin[-\alpha, \alpha],
\end{array} \quad \alpha>0 .\right.
\end{gathered}
$$

The set $\mathcal{U}=(0, \infty)$ for $\left(f_{\alpha}, \alpha>0\right)$ is directed in the descending order, while for $\left(\varphi_{\alpha}, \alpha>0\right)$ it is in the ascending order.

Definition 6. Given a directed set $\mathcal{U}$, a bounded net $\left(e_{\alpha}, \alpha \in \mathcal{U}\right)$ of functions from $L^{1}(\mathbb{R})$ is called the bounded approximate identity (BAI) of $L^{1}(\mathbb{R})$ if the conditions below are met:

1) $\widehat{e}_{\alpha}(0)=1$ for all $\alpha \in \mathcal{U}$;

2) $\lim _{\alpha} e_{\alpha} * f=f$ for all $f$ from $L^{1}(\mathbb{R})$.

Definition 7. Given a directed set $\mathcal{U}$, a bounded net $\left(f_{\alpha}, \alpha \in \mathcal{U}\right)$ of functions from $L^{1}(\mathbb{R})$ is called an invariant integral if the conditions below are true:

1) $\widehat{f}_{\alpha}(0)=1$ and $f_{\alpha} \geqslant 0$ for all $\alpha \in \mathcal{U}$;

2) $\lim _{\alpha} \int_{\mathbb{R}}\left|f_{\alpha}(t+u)-f_{\alpha}(t)\right| d t=0$ for all $u \in \mathbb{R}$.

Below we use the following concept of an almost periodic vector in a Banach space $\mathcal{X}$ (see $[2,5,6]$ ), carrying a strongly continuous isometric representation $T: \mathbb{R} \rightarrow$ End $\mathcal{X}$.

Definition 8. A vector $x_{0} \in \mathcal{X}$ is called almost periodic (with respect to representation $T$ ) if one of the following conditions is met:

1) for every $\varepsilon>0$ the set $\Omega\left(\varepsilon, x_{0}\right)=\left\{\omega \in \mathbb{R}:\left\|T(\omega) x_{0}-x_{0}\right\|<\varepsilon\right\}$ of $\varepsilon$-periods of the vector $x_{0}$ is relatively dense in $\mathbb{R}$;

2) the orbit $\left\{T(t) x_{0}, t \in \mathbb{R}\right\}$ of $x_{0}$ is precompact in $\mathcal{X}$;

3) $t \mapsto \varphi(t)=T(t) x_{0}, t \in \mathbb{R}$, is a continuous almost periodic function, i. e., $\varphi \in A P(\mathbb{R}, X)$ (see $[5,15])$;

4) for any $\varepsilon>0$ there are eigenvalues $\lambda_{1}, \ldots, \lambda_{N}$ and associated eigenvectors $x_{1}, \ldots, x_{N}$ of the representation $T$, i. e., $T(t) x_{k}=e^{i \lambda_{k} t} x_{k}$ for $t \in \mathbb{R}$ and $k=1, \ldots, N$ such that

$$
\left\|x_{0}-\sum_{k=1}^{N} x_{k}\right\|<\varepsilon .
$$


The set $A P(\mathcal{X})=A P(\mathcal{X}, T)$ of almost periodic vectors (with respect to a representation $T)$ is a closed submodule of $\mathcal{X}$. Observe that $A P\left(C_{b, u}(\mathbb{R}, X), S\right)=A P(\mathbb{R}, X)$ and $A P(\mathcal{X}) \subset \mathcal{X}_{c}$ (see $\left.[3,4,7]\right)$.

A unique linear operator $\mathcal{J} \in \operatorname{End} A P(\mathcal{X})$ with the properties

1) $\|\mathcal{J}\|=1$;

2) $\mathcal{J}(T(t) x)=\mathcal{J} x$ for $t \in \mathbb{J}$ and $x \in \mathcal{X}$;

exists on a Banach space $A P(\mathcal{X})$ of almost periodic vectors. Consider a function $\widehat{x}_{B}: \mathbb{R} \rightarrow \mathcal{X}, \widehat{x}_{B}(\lambda)=\mathcal{J}\left(T_{\lambda} x\right)$, where $T_{\lambda}(t)=T(t) e^{-i \lambda t}$ for $t \in \mathbb{R}$, for every vector $x$ from $A P(\mathcal{X})$. This function is called the Bohr transform of the vector $x$. Its support $\operatorname{supp} \widehat{x}_{B}$ is at most a countable set, i. e., $\operatorname{supp} \widehat{x}_{B}=\left\{\lambda_{1}, \lambda_{2}, \ldots\right\}$, and

$$
T(t) x_{k}=e^{i \lambda_{k} t} x_{k}, t \in \mathbb{R}, k \geqslant 1,
$$

where $x_{k}, k \geqslant 1$, are eigenvectors of the representation $T$; they are also eigenvectors of the generator $i A$ of the operator group $T$, i.e., $i A x_{k}=$ $=i \lambda_{k} x_{k}, k \geqslant 1$. Moreover, $\Lambda\left(x_{k}\right)=\left\{\lambda_{k}\right\}$ for $k \geqslant 1$. The set $\Lambda_{B}(x)=$ $=\left\{\lambda_{1}, \lambda_{2}, \ldots\right\}$ is called the Bohr spectrum of the vector $x \in A P(\mathcal{X})$. Note that

$$
\begin{gathered}
\widehat{x}_{B}(\lambda)=\lim _{\alpha \rightarrow \infty} \frac{1}{\alpha} \int_{0}^{\alpha} T(\tau) x e^{-i \lambda \tau} d \tau=\lim _{0<\varepsilon \rightarrow 0} \varepsilon \int_{0}^{\varepsilon^{-1}} T(\tau) x e^{-(\varepsilon+i \lambda) \tau} d \tau= \\
=\lim _{0<\varepsilon \rightarrow 0} \varepsilon R(\varepsilon+i \lambda, i A) x=\lim _{\alpha} f_{\alpha} x, \lambda \in \mathbb{R},
\end{gathered}
$$

where $\left(f_{\alpha}, \alpha \in \mathcal{U}\right)$ is an arbitrary $\lambda$-net from $L^{1}(\mathbb{R})$.

The series

$$
x \sim \sum_{k \geqslant 1} x_{k}
$$

is called the Fourier series of the vector $x \in A P(\mathcal{X})$ and $x_{k}, k \geqslant 1$, are called the Fourier coefficients of $x$. Note that if the series absolutely converges, then $x=\sum_{k \geqslant 1} x_{k}$.

Note the uniqueness property of the Bohr transform: if the Bohr transform $\widehat{x}_{B}$ of a vector $x \in A P(\mathcal{X})$ is equal to zero, then $x=0$.

Lemma 2. [13] For any function $f$ from $L^{1}(\mathbb{R})$ and any almost periodic vector $x \in A P(\mathcal{X})$ with the Fourier series (3), the vector $f x$ is almost periodic with the Fourier series of the form

$$
f x \sim \sum_{k \geqslant 1} \widehat{f}\left(\lambda_{k}\right) x_{k} .
$$


The following lemma uses the (BAI) $\left(f_{n}, n \geqslant 1\right)$ in $L^{1}(\mathbb{R})$ (see Definition 6) constructed below (see $[2,5,6])$.

Let us consider a function $\widehat{f}_{0}$ from $\widehat{L}^{1}(\mathbb{R})$ of Fourier transforms of functions from $L^{1}(\mathbb{R})$ (with pointwise multiplication) with compact support supp $\widehat{f}_{0}$ on the interval $[-1,1]$, such that $\widehat{f}_{0}(0)=1$. Then the sequence $\left(f_{n}\right), n \geqslant 1$, of functions defined by $f_{n}(t)=n f_{0}(n t), t \in \mathbb{R}$, is a (BAI) in $L^{1}(\mathbb{R})$. Note that $\left\|f_{n}\right\|=\left\|f_{0}\right\|$ for $n \geqslant 1$.

Lemma 2 implies

Lemma 3. [13] Let $x$ be an almost periodic vector from $A P(\mathcal{X})$ with the Bohr spectrum $\Lambda_{B}(x)=\left\{\lambda_{1}, \lambda_{2}, \ldots\right\}$ satisfying the condition $\lim _{n \rightarrow \infty}\left|\lambda_{n}\right|=\infty$. In this case

$$
\lim _{n \rightarrow \infty}\left\|x-f_{n} x\right\|=\lim _{n \rightarrow \infty}\left\|x-\sum_{\left|\lambda_{k}\right|<n} \widehat{f}_{0}\left(\frac{\lambda_{k}}{n}\right) x_{k}\right\|,
$$

where $x_{k}, k \geqslant 1$, are the Fourier coefficients of $x$.

Lemma 4. The Bohr transform $\widehat{x}_{B}: \mathbb{R} \rightarrow \mathcal{X}$ of a vector $x$ from $A P(\mathcal{X})$ can be estimated as follows

$$
\left\|\widehat{x}_{B}(\lambda)\right\| \leqslant\|x\|, \lambda \in \mathbb{R} .
$$

Proof. Given a directed set $\mathcal{U}$, in the equality $\widehat{x}_{B}(\lambda)=\lim f_{\alpha} x, x \in A P(\mathcal{X})$, we should use a $\lambda$-net $\left(f_{\alpha}, \alpha \in \mathcal{U}\right)$ from $L^{1}(\mathbb{R})$, which is an invariant integral (see Definition 7). In this case $\left\|f_{\alpha}\right\|=\widehat{f}_{\alpha}(0)=1$ for any $\alpha \in \mathcal{U}$ and, consequently, $\left\|\widehat{x}_{B}(\lambda)\right\| \leqslant \sup \left\|f_{\alpha}\right\|\|x\|=\|x\|, \lambda \in \mathbb{R}$.

Theorem 1. For any vector $x$ from $A P(\mathcal{X})$ with the Bohr spectrum $\Lambda_{B}(x)=\left\{\lambda_{1}, \lambda_{2}, \ldots\right\}$ the equality $\lim _{n \rightarrow \infty}\left\|\widehat{x}_{B}\left(\lambda_{n}\right)\right\|=0$ holds true.

Proof. Condition 4) of Definition 8 implies that there exists a sequence $\left(x_{n}, n \in \mathbb{N}\right)$ of vectors from $A P(\mathcal{X})$ with finite Bohr transform supports $\operatorname{supp} \widehat{\left(x_{n}\right)_{B}}, n \geqslant 1$. Lemma 4 implies that $\sup \left\|\widehat{x}_{B}(\lambda)-\widehat{\left(x_{n}\right)_{B}}(\lambda)\right\| \rightarrow 0$, $n \rightarrow \infty$. Hence, $\lim _{n \rightarrow \infty}\left\|\widehat{x}_{B}\left(\lambda_{n}\right)\right\|=0$.

$$
\lambda \in \mathbb{R}
$$

Given $m>0$ and $x \in A P(\mathcal{X})$, consider

$$
\tau_{m}(x)=\sum_{\left|\lambda_{k}\right|<m, \lambda_{k} \in \Lambda_{B}(x)}\left(1-\frac{\left|\lambda_{k}\right|}{m}\right) x_{k},
$$

where $\Lambda_{B}(x)=\left\{\lambda_{1}, \lambda_{2}, \ldots\right\}$ and $x_{k}$ with $k \geqslant 1$ are the Fourier coefficients of $x$ (see (3)). 
Theorem 2. [15] The Fourier series (3) of a vector $x$ from $A P(\mathcal{X})$ is summable by the Bochner-Feier method, i.e., the equality $\lim _{m \rightarrow \infty}\left\|x-\tau_{m}(x)\right\|=0$ holds.

If $\mathcal{X}_{0}$ is a closed submodule of $\mathcal{X}$ invariant under operators $T(t), t \in \mathbb{R}$, then the quotient space $\mathcal{X} / \mathcal{X}_{0}$ is also a Banach $L^{1}(\mathbb{R})$-module, whose structure for all $f \in L^{1}(\mathbb{R})$ and equivalence classes $\widetilde{x}=x+\mathcal{X}_{0}, x \in \mathcal{X}$, is defined as $f \widetilde{x}=f x+\mathcal{X}_{0}=\widetilde{f x}$. This structure is associated with the representation $\widetilde{T}: \mathbb{R} \rightarrow$ End $\mathcal{X} / \mathcal{X}_{0}, \quad \widetilde{T}(t) \widetilde{x}=\widetilde{T(t) x}=T(t) x+\mathcal{X}_{0}, x \in \mathcal{X}$.

Denote the quotient space $\mathcal{F}(\mathbb{J}, X) / \mathcal{F}_{0}(\mathbb{J}, X)$ by $\mathcal{X}(\mathbb{J}), \mathbb{J} \in\left\{\mathbb{R}_{+}, \mathbb{R}\right\}$; this is a Banach space under the norm $\|\widetilde{x}\|=\inf _{y \in x+\mathcal{F}_{0}}\|y\|$, where $\widetilde{x}=x+$ $+\mathcal{F}_{0}(\mathbb{I}, X)$ is the equivalence class that contains the function $x \in \mathcal{F}(\mathbb{J}, X)$. A Banach space $\mathcal{X}(\mathbb{J})$ is a Banach algebra with the multiplication rule defined by $\widetilde{x} \widetilde{y}=\widetilde{x y}, \widetilde{x}, \widetilde{y} \in \mathcal{X}(\mathbb{J})$.

Define the strongly continuous group of isometries $\widetilde{S}: \mathbb{R} \rightarrow \operatorname{End} \mathcal{X}(\mathbb{R})$ on $\mathcal{X}(\mathbb{R})$ by the formula

$$
\widetilde{S}(t) \widetilde{x}=\widetilde{S(t) x}, x \in \mathcal{F}(\mathbb{R}, X), t \in \mathbb{R} .
$$

The quotient space $\mathcal{X}(\mathbb{R})$ is endowed with the structure of a Banach $L^{1}(\mathbb{R})$ module using the formula $f \widetilde{x}=\widetilde{f * x}, f \in L^{1}(\mathbb{R}), x \in \mathcal{F}(\mathbb{R}, X)$.

Remark 3. Assume that $\mathbb{J}=\mathbb{R}_{+}$. Each function $x \in \mathcal{F}\left(\mathbb{R}_{+}, X\right)$ can be extended to the function $y$ on $\mathbb{R}$, so that $y$ satisfied all five conditions of Definition 2. Note that the equivalence class $\widetilde{x} \in \mathcal{X}(\mathbb{R})$ does not depend on the certain extension and, consequently, the Banach space $\mathcal{X}\left(\mathbb{R}_{+}\right)$is isometrically embedded in $\mathcal{X}(\mathbb{R})$ as a closed submodule. In this case, the group $\widetilde{S}$ is well-defined on $\mathcal{X}\left(\mathbb{R}_{+}\right)$.

3. Almost periodic at infinity functions. Consider a homogeneous function space $\mathcal{F}(\mathbb{J}, X)$ satisfying the conditions (a)-(d) from Definition 1 for $\mathbb{J}=\mathbb{R}$ (the conditions 1 ) - 5) from Definition 2 for $\mathbb{J}=\mathbb{R}_{+}$). Consider the (semi-)group $S: \mathbb{J} \rightarrow \operatorname{End} \mathcal{F}(\mathbb{J}, X)$ of operators of the form

$$
(S(t) x)(\tau)=x(t+\tau), t, \tau \in \mathbb{J}
$$

in a Banach space $\mathcal{F}(\mathbb{J}, X)$.

Definition 9. A function $x \in \mathcal{F}_{c}(\mathbb{J}, X)$ is called slowly varying at infinity if and only if the condition $S(\alpha) x-x \in \mathcal{F}_{0}(\mathbb{J}, X)$ is fulfilled for every $\alpha \in \mathbb{J}$. 
Denote the set of slowly varying at infinity functions from $\mathcal{F}(\mathbb{J}, X)$ by $\mathcal{F}_{s l, \infty}(\mathbb{J}, X)$. Definition 9 directly implies that $\mathcal{F}_{s l, \infty}(\mathbb{J}, X)$ is a closed subspace of $\mathcal{F}(\mathbb{J}, X)$ that is invariant under the shift operators $S(t), t \in \mathbb{J}$. Slowly varying and periodic at infinity functions from homogeneous spaces were studied in [16].

In the case $\mathcal{F}(\mathbb{J}, X)=C_{b, u}(\mathbb{J}, X)$ the above definition is equivalent to the classical definition of continuous slowly varying at infinity function (see $[3,4,8])$. The set of these functions is denoted by $C_{s l, \infty}(\mathbb{J}, X)$. Particularly, the solutions of the heat equation were established to belong to $C_{s l, \infty}(\mathbb{J}, X)$ in [8].

Lemma 5. For every $x \in \mathcal{F}_{s l, \infty}(\mathbb{R}, X)$ there exists a function $x_{0} \in C_{s l, \infty}(\mathbb{R}, X)$ such that $x-x_{0} \in \mathcal{F}_{0}(\mathbb{R}, X)$. Moreover, for every $x \in \mathcal{F}_{s l, \infty}(\mathbb{R}, X)$ there is a function $y: \mathbb{R} \rightarrow X$ that can be extended on $\mathbb{C}$ to a bounded entire function of the exponential type and such that $y-x \in \mathcal{F}_{0}(\mathbb{R}, X)$.

For $\mathbb{J}=\mathbb{R}$ this result was proved in [16]. The result for $\mathbb{J}=\mathbb{R}_{+}$follows from Remark 3.

For example, a function $x=c+x_{0} \in \mathcal{F}(\mathbb{J}, X)$, where $c$ is a vector from $X$ and $x_{0}$ is a function from $\mathcal{F}_{0}(\mathbb{J}, X)$, belongs to $\mathcal{F}_{s l, \infty}(\mathbb{J}, X)$.

Let us give four definitions of almost periodic at infinity functions from the homogeneous space $\mathcal{F}(\mathbb{J}, X)$. After that, we are going to prove them to be equivalent and study their Fourier series.

First, let us introduce a definition of a continuous almost periodic at infinity function (see $[3,4]$ ) that is based on the notion of $\varepsilon$-period at infinity.

Definition 10. Assume $\varepsilon>0$. A number $\omega>0$ is called an $\varepsilon$-period at infinity of $x \in C_{b}(\mathbb{J}, X)$ if there exists a number $a(\varepsilon)>0$ such that

$$
\sup _{|t| \geqslant a(\varepsilon)}\|x(t+\omega)-x(t)\|<\varepsilon .
$$

Denote the set of $\varepsilon$-periods at infinity of a function $x \in C_{b}(\mathbb{J}, X)$ by $\Omega_{\infty}(\varepsilon, x)$.

Definition 11. A subset $\Omega$ of $\mathbb{J}$ is called relatively dense on $\mathbb{J}$ if there exists an $l>0$ with $[t, t+l] \cap \Omega \neq \varnothing$ for every $t \in \mathbb{J}$.

Definition 12. A function $x \in C_{b, u}(\mathbb{J}, X)$ is called almost periodic at infinity if for every $\varepsilon>0$ the set $\Omega_{\infty}(\varepsilon, x)$ of its $\varepsilon$-periods at infinity is relatively dense on $\mathbb{J}$. 
The set of almost periodic at infinity functions from $C_{b, u}(\mathbb{J}, X)$ is denoted by $A P_{\infty}(\mathbb{J}, X)$ and studied in $[3,4,11,13]$. Definitions 10 and $12 \mathrm{imp}-$ ly that every function $x \in C_{b, u}(\mathbb{R}, X)$ almost periodic in the Bohr sense $(x \in A P(\mathbb{R}, X)$, see $[5,15])$ is almost periodic at infinity. By $A P\left(\mathbb{R}_{+}, X\right)$ denote the set of almost periodic Bohr functions that are restrictions to $\mathbb{R}_{+}$of functions from $A P(\mathbb{R}, X)$. Therefore, $A P(\mathbb{J}, X) \subset A P_{\infty}(\mathbb{J}, X)$.

Definition 13. Assume that $\varepsilon>0$. A number $\omega>0$ is called an $\varepsilon$-period at infinity of $x \in \mathcal{F}(\mathbb{J}, X)$ if there exists a function $x_{0} \in \mathcal{F}_{0}(\mathbb{J}, X)$ such that

$$
\left\|S(\omega) x-x-x_{0}\right\|<\varepsilon .
$$

For the set of $\varepsilon$-periods at infinity of $x \in \mathcal{F}(\mathbb{J}, X)$ we use the same denotation $\Omega_{\infty}(\varepsilon, x)$.

Definition 14. A function $x$ from $\mathcal{F}_{c}(\mathbb{J}, X)$ is called almost periodic at infinity if for any $\varepsilon>0$ the set $\Omega_{\infty}(\varepsilon, x)$ of its $\varepsilon$-periods at infinity is relatively dense on $\mathbb{J}$.

Denote the set of functions from $\mathcal{F}(\mathbb{J}, X)$ almost periodic at infinity by $A P_{\infty} \mathcal{F}(\mathbb{I}, X)$. Note that $A P_{\infty} \mathcal{F}(\mathbb{J}, X)$ is a closed subspace of $\mathcal{F}(\mathbb{J}, X)$ invariant under the shift operators $S(t), t \in \mathbb{J}$. Definition 9 directly implies that $\Omega_{\infty}(\varepsilon, x)=\mathbb{J}$ for any $x \in \mathcal{F}_{s l, \infty}(\mathbb{J}, X)$ and $\varepsilon>0$, hence, $x \in A P_{\infty} \mathcal{F}(\mathbb{J}, X)$. Consequently, $\mathcal{F}_{s l, \infty}(\mathbb{J}, X) \subset A P_{\infty} \mathcal{F}(\mathbb{J}, X)$.

In the case $\mathcal{F}(\mathbb{I}, X)=C_{b, u}(\mathbb{J}, X)$ the above definition is equivalent to Definition 12.

Definition 15. A set of functions $\mathcal{M} \subset \mathcal{F}(\mathbb{J}, X)$ is called precompact at infinity if for any $\varepsilon>0$ there exist finitely many functions $b_{1}, \ldots, b_{N} \in \mathcal{M}$ called an $\varepsilon$-net at infinity, such that for every $x \in \mathcal{M}$ there exists a function $b_{k}, 1 \leqslant k \leqslant N$, and a function $\alpha_{\varepsilon} \in \mathcal{F}_{0}(\mathbb{J}, X)$ such that

$$
\left\|x-b_{k}-\alpha_{\varepsilon}\right\|<\varepsilon \text {. }
$$

Definition 16. A function $x \in \mathcal{F}_{c}(\mathbb{J}, X)$ is called almost periodic at infinity if the set $\mathcal{M}=\{S(k) x, k \in \mathbb{J}\}$ is precompact at infinity.

For $\mathcal{F}(\mathbb{J}, X)=C_{b, u}(\mathbb{I}, X)$ Definition 16 corresponds to the Bochner's criterion (see [15]) of almost periodicity.

Observe that the functions of the form

$$
x(t)=\sum_{k=1}^{N} x_{k}(t) e^{i \lambda_{k} t}, x_{1}, \ldots, x_{N} \in \mathcal{F}_{s l, \infty}(\mathbb{J}, X), \lambda_{1}, \ldots, \lambda_{N} \in \mathbb{R}, t \in \mathbb{J},
$$


called generalized trigonometric polynomials, are almost periodic at infinity in the sense of Definition 16.

Definition 17. A function $x \in \mathcal{F}_{c}(\mathbb{J}, X)$ is called almost periodic at infinity if, given $\varepsilon>0$, we can indicate a finite collection $\lambda_{1}, \ldots, \lambda_{N}$ of real numbers and functions $x_{1}, \ldots, x_{N}$ from $\mathcal{F}_{s l, \infty}(\mathbb{J}, X)$ such that

$$
\left\|x-\sum_{k=1}^{N} x_{k} e_{k}\right\|<\varepsilon,
$$

where $e_{k}, 1 \leqslant k \leqslant N$, are functions defined by $e_{k}(t)=e^{i \lambda_{k} t}, t \in \mathbb{R}$.

Definition 18. A function $x$ from $\mathcal{F}_{c}(\mathbb{J}, X)$ is called almost periodic at infinity if the equivalence class $\widetilde{x}=x+\mathcal{F}_{0}(\mathbb{J}, X)$ is an almost periodic vector from $\mathcal{X}(\mathbb{J})=\mathcal{F}(\mathbb{J}, X) / \mathcal{F}_{0}(\mathbb{J}, X)$ with respect to the isometric representation $\widetilde{S}: \mathbb{R} \rightarrow$ End $\mathcal{X}$.

The almost periodic at infinity functions from $C_{b, u}(\mathbb{J}, X)$ appeared, for the first time, in $[3,4]$. In these articles the definition corresponding to Definition 18 was used. The main results of those articles deal with the asymptotic behaviour of bounded operator semigroups.

Theorem 3. All definitions of almost periodic at infinity functions from $\mathcal{F}(\mathbb{J}, X)$ (Definitions 14, 16, 17, 18) are equivalent.

Proof. We assume that $\mathbb{J}=\mathbb{R}$ (the result for $\mathbb{J}=\mathbb{R}_{+}$follows from Remark 3). Let us consider the quotient space $\mathcal{X}=\mathcal{F}(\mathbb{R}, X) / \mathcal{F}_{0}(\mathbb{R}, X)$ and the group of isometries $T=\widetilde{S}: \mathbb{R} \rightarrow$ End $\mathcal{X}$ defined above. For this representation, Definition 17 corresponds to property 4) of Definition 8. Since all of the properties of Definition 8 are equivalent, it suffices to show that the first three properties are equivalent to Definitions 14, 16, and 17, respectively.

Given $x \in \mathcal{F}(\mathbb{R}, X)$, take the equivalence class $\widetilde{x}$ in $\mathcal{X}$, constructed from $x$. Then for each $\varepsilon>0$ the set $\Omega_{\infty}(\varepsilon, x) \cup\left(-\Omega_{\infty}(\varepsilon, x)\right)$ coincides with the set $\Omega(\varepsilon, \widetilde{x})$ of $\varepsilon$-periods of $\widetilde{x}$. Hence, the corresponding definitions are equivalent.

The equivalence of Definition 16 and property 2) of Definition 8 follows directly from the definition of the quotient module $\mathcal{X}$.

In order to verify the equivalence of Definition 17 and property 4) of Definition 8, it suffices to establish that the Beurling spectrum $\Lambda(\widetilde{y})$ of the equivalence class $\widetilde{y} \in \mathcal{X}$ with $\widetilde{y}=y+\mathcal{F}_{0}(\mathbb{R}, X)$ is the singleton 
$\left(\Lambda(\widetilde{y})=\left\{\lambda_{0}\right\}\right)$ if and only if $y \in \mathcal{F}(\mathbb{R}, X)$ can be represented in the form $y(t)=y_{0}(t) e^{i \lambda_{0} t}$ for $t \in \mathbb{R}$, where $y_{0} \in \mathcal{F}_{s l, \infty}(\mathbb{R}, X)$.

If $\Lambda(\widetilde{y})=\left\{\lambda_{0}\right\}$, then $\widetilde{S}(t) \widetilde{y}=e^{i \lambda_{0} t} \widetilde{y}$ for every $t \in \mathbb{R}$ (see property 4) of Lemma 1). Hence, $\Lambda\left(\widetilde{y}_{0}\right)=\{0\}$, where $y_{0}(s)=y(s) e^{-i \lambda_{0} s}$ for $s \in \mathbb{R}$. Therefore, $\widetilde{S}(t) \widetilde{y}_{0}=\widetilde{y}_{0}$ for every $t \in \mathbb{J}$. Thus, $S(t) y_{0}-y_{0} \in \mathcal{F}_{0}(\mathbb{R}, X)$ for $t \in \mathbb{R}$, i. e., $y_{0} \in \mathcal{F}_{s l, \infty}(\mathbb{R}, X)$.

Conversely, if $y(t)=y_{0}(t) e^{i \lambda_{0} t}$ for $t \in \mathbb{R}$, where $y_{0} \in \mathcal{F}_{0}(\mathbb{J}, X)$, then $\widetilde{S}(t) \widetilde{y}=e^{i \lambda_{0} t} \widetilde{y}$ for $t \in \mathbb{R}$, and so property 4) of Lemma 1 implies that $\Lambda(\widetilde{y})=\left\{\lambda_{0}\right\}$ (see Remark 2).

Given $x \in A P_{\infty} \mathcal{F}(\mathbb{I}, X)$, let us consider the series

$$
\widetilde{x} \sim \sum_{n \geqslant 1} \widetilde{y}_{n}, \Lambda_{B}(\widetilde{x})=\left\{\lambda_{1}, \lambda_{2}, \ldots\right\}, \Lambda\left(\widetilde{y}_{n}\right)=\lambda_{n}
$$

which is the Fourier series of the equivalence class $\widetilde{x}=x+\mathcal{F}_{0}(\mathbb{J}, X) \in$ $A P(\mathcal{X})$.

Definition 19. The series

$$
x(t) \sim \sum_{n \geqslant 1} x_{n}(t) e^{i \lambda_{n} t}, t \in \mathbb{J},
$$

where functions $z_{n}, n \geqslant 1$, of the form $z_{n}(t)=x_{n}(t) e^{i \lambda_{n} t}, t \in \mathbb{J}, x_{n} \in$ $\mathcal{F}_{c}(\mathbb{J}, X)$, belong to the corresponding equivalence classes $\widetilde{y}_{n}$ with $n \geqslant 1$, is called the Fourier series of $x$. The functions $x_{n}$ with $n \geqslant 1$ are called the Fourier coefficients of $x$.

Note that the Fourier series defined this way is ambiguous, i. e., the functions $x_{n}$ with $n \geqslant 1$ can be chosen differently. In [3,4] the analogous definition was given for functions from $C_{b, u}(\mathbb{J}, X)$.

Theorem 4. Coefficients of any Fourier series (4) of a function $x \in A P_{\infty} \mathcal{F}(\mathbb{J}, X)$ belong to $\mathcal{F}_{s l, \infty}(\mathbb{J}, X)$ and satisfy the condition $\lim _{n \rightarrow \infty}\left\|x_{n}\right\|_{\mathcal{F}}=0$.

The condition $x_{n} \in \mathcal{F}_{s l, \infty}(\mathbb{J}, X)$ follows from Definition 17 and the equality $\lim _{n \rightarrow \infty}\left\|x_{n}\right\|_{\mathcal{F}}=0$ follows from Theorem 1 .

Theorem 4 and Lemma 5 directly imply

Theorem 5. Given a function $x \in A P_{\infty} \mathcal{F}(\mathbb{J}, X)$, one can construct the Fourier series (4) such that $x_{n} \in C_{s l, \infty}(\mathbb{J}, X), n \geqslant 1$. Moreover, the 
functions $x_{n}$ with $n \geqslant 1$ can be extended on $\mathbb{C}$ to bounded entire functions of the exponential type.

Given $m>0$ and $x \in A P_{\infty} \mathcal{F}(\mathbb{J}, X)$, consider a function $\tau_{m}(x, \cdot): \mathbb{J} \rightarrow X$ defined by

$$
\tau_{m}(x, t)=\sum_{\left|\lambda_{n}\right|<m, \lambda_{n} \in \Lambda_{B}(\widetilde{x})}\left(1-\frac{\left|\lambda_{n}\right|}{m}\right) x_{k}(t) e^{i \lambda_{n} t}, t \in \mathbb{J},
$$

where $x_{k}, k \geqslant 1$, are the Fourier coefficients of $x$.

Definition 20. The Fourier series (4) of a function $x \in A P_{\infty} \mathcal{F}(\mathbb{J}, X)$ is summable at infinity by the Bochner-Feier method, if a sequence $\left(y_{m}^{0}\right.$, $m \in \mathbb{N})$ of functions from $\mathcal{F}_{0}(\mathbb{J}, X)$ such that

$$
\lim _{m \rightarrow \infty}\left\|x-\tau_{m}(x, \cdot)-y_{m}^{0}\right\|_{\mathcal{F}}=0
$$

exists.

For $\mathcal{F}(\mathbb{J}, X)=C_{b, u}(\mathbb{J}, X)$ condition (5) is equivalent to

$$
\lim _{m \rightarrow \infty} \sup _{t \in \mathbb{J}}\left\|x(t)-\tau_{m}(x, t)-y_{m}^{0}(t)\right\|_{X}=0 .
$$

Theorem 6. The Fourier series (4) of a function $x \in A P_{\infty} \mathcal{F}(\mathbb{J}, X)$ is summable at infinity by the Bochner-Feier method.

Proof. Assume that $\mathbb{J}=\mathbb{R}$ (the result for $\mathbb{J}=\mathbb{R}_{+}$follows from Remark 3). Let us consider the quotient space $\mathcal{X}=\mathcal{F}(\mathbb{R}, X) / \mathcal{F}_{0}(\mathbb{R}, X)$ and the group of isometries $T=\widetilde{S}: \mathbb{R} \rightarrow$ End $\mathcal{X}$ defined above. For a function $x \in A P_{\infty} \mathcal{F}(\mathbb{R}, X)$ an equivalence class $\widetilde{x}$ belongs to $A P(\mathcal{X})$. Therefore, it satisfies the conditions of Theorem 2. The statement of Theorem 6 follows directly from Theorem 2 .

Note that the choice of Fourier coefficients in the last theorem is not essential.

Acknowledgment. The first author of this work was supported by RFBR according to the research project 16-01-00197, the second author was supported by RFBR according to the research project 18-31-00097 and the third author was supported by RFBR according to the research project 18-31-00097. 


\section{References}

[1] Arendt. W., Batty C. J. K., Hieber M., Neubrander F. Vector-valued Laplace transforms and Cauchy problems. Basel, Birkhäuser, Monographs in Mathematics, 2011, Vol. 96.

[2] Baskakov A. G. Harmonic Analysis in Banach Modules and the Spectral Theory of Linear Operators. Izdat. Dom VGU, Voronezh, 2016.

[3] Baskakov A. G. Harmonic and spectral analysis of power bounded operators and bounded semigroups of operators on Banach spaces. Math. Notes, 2015, vol. 97, no. 2, pp. 164-178. DOI: https://doi.org/10.4213/mzm10285.

[4] Baskakov A. G. Analysis of linear differential equations by methods of the spectral theory of difference operators and linear relations. Russian Math. Surveys, 2013, vol. 68, no. 1, pp. 69-116. DOI: https://doi.org/10. $4213 /$ rm9505.

[5] Baskakov A. G. Representation theory for Banach algebras, Abelian groups, and semigroups in the spectral analysis of linear operators. J. Math. Sci., 2006, vol. 137, no. 4, pp. 4885-5036. DOI: https://doi.org/10.1007/ s10958-006-0286-4.

[6] Baskakov A. G. Spectral criteria for almost periodicity of solutions of functional equations. Math. Notes, 1978, vol. 24, no. 2, pp. 606-612. DOI: https://doi.org/10.1007/BF01105312.

[7] Baskakov A. G. Some Problems of the Theory of Vector Almost Periodic Functions. Diss. Kand. Fiz.-Mat. Nauk, Voronezh, 1973.

[8] Baskakov A. G., Kaluzhina N. S. Beurling's theorem for functions with essential spectrum from homogeneous spaces and stabilization of solutions of parabolic equations. Math. Notes, 2012, vol. 92, no. 5, pp. 587-605. DOI: https://doi.org/10.4213/mzm8963.

[9] Baskakov A. G., Krishtal I. A. Spectral analysis of abstract parabolic operators in homogeneous function spaces. Mediterranean Journal of Mathematics, 2016, vol. 13, no. 5, pp. 2443-2462. DOI: https://doi.org/10. 1007/s00009-015-0633-0.

[10] Baskakov A. G., Krishtal I. A. Harmonic analysis of causal operators and their spectral properties. Izv. Math., 2005, vol. 69, no. 3, pp. 439-486. DOI: https://doi.org/10.4213/im639.

[11] Baskakov A., Obukhovskii V., Zecca P. Almost periodic solutions at infinity of differential equations and inclusions. Siberian Math. J., 2018, vol. 462, no. 1, pp. 747-763. DOI: https://doi.org//10.1016/j.jmaa.2018.02. 034. 
[12] Baskakov A., Strukova I. Harmonic analysis of functions periodic at infinity. Eurasian Math. J., 2016, vol. 7, no. 4, pp. 9-29.

[13] Baskakov A. G., Strukova I. I., Trishina I. A. Solutions almost periodic at infinity to differential equations with unbounded operator coefficients. Siberian Math. J., 2018, vol. 55, no. 2, pp. 231-242. https://doi.org/ DOI : 10.17377/smzh.2018.59.205.

[14] Hewitt E., Ross K. Abstract Harmonic Analysis. Vol. 2, Springer-Verlag, New York, 1975.

[15] Levitan B. M., Zhikov V. V. Almost-Periodic Functions and Differential Equations. Izdat. MGU, Moscow, 1978.

[16] Strukova I. I. Harmonic analysis of periodic at infinity functions in homogeneous spaces. Science Journal of Volgograd State University. Mathematics. Physics, 2017, vol. 39, no. 2, pp. 29-38. DOI: https://doi.org/10.15688/ jvolsu1.2017.2.3.

[17] Strukova I. I. On Wiener's theorem for functions periodic at infinity. Siberian Math. J., 2016, vol. 57, no. 1, pp. 145-154. DOI: https://doi. org/10.17377/smzh.2016.57.114.

[18] Strukova I. I. Spectra of algebras of slowly varying and periodic at infinity functions and Banach limits. Proc. Voronezh State Univ. Ser. Physics. Mathematics, 2015, no. 3, pp. 161-165.

[19] Strukova I. I. Harmonic analysis of periodic vectors and functions periodic at infinity. J. Math. Sci., 2015, vol. 211, no. 6, pp. 874-885. DOI: https: //doi.org/10.1007/s10958-015-2641-9.

Received July 8, 2018.

In revised form, December 3, 2018.

Accepted December 4, 2018.

Published online December 19, 2018.

Voronezh State University

1 Universitetskaya sq., Voronezh 394006, Russia

E-mails:

Baskakov A. G. anatbaskakov@yandex.ru, Strukov V. E. sv.post.of.chaos@gmail.com, Strukova I. I. irina.k.post@yandex.ru 